\title{
Antiplasmodial Efficacy of Crude Cocoa Powder Extract on CD4+ T-Cell Counts of Plasmodium berghei Infected BALB/c Mice
}

\author{
Ololade Dolapo Aladesemipe ${ }^{1 *}$, Bakarey Adeleye Solomon ${ }^{1}$, Oladosu Ibrahim ${ }^{2}$, Oluwadun Afolabi ${ }^{3}$ \\ ${ }^{1}$ Institute for Advanced Medical Research and Training, College of Medicine, University of Ibadan, Ibadan, Nigeria \\ ${ }^{2}$ Department of Chemistry, University of Ibadan, Ibadan, Nigeria \\ ${ }^{3}$ Department of Medical Microbiology and Parasitology, Olabisi Onabanjo University, Ogun State, Nigeria \\ Email: ${ }^{1}$ loladealade2000@yahoo.ca
}

Received June 14, 2013; revised July 14, 2013; accepted July 22, 2013

Copyright (C) 2013 Ololade Dolapo Aladesemipe et al. This is an open access article distributed under the Creative Commons Attribution License, which permits unrestricted use, distribution, and reproduction in any medium, provided the original work is properly cited.

\begin{abstract}
Background: Drug resistance in malaria warrants the need for alternative therapy from plant food nutrients. The search for novel anti-malarial control spurred a great interest in cocoa which has been portrayed as immune booster against malaria. This study was geared towards estimation of CD4+ cells of $P$. berghei infected mice treated with cocoa powder extract (CPE) to provide substantive scientific evidence to authenticate the anecdotal report. Methods: Brine shrimp toxicity assay was done to determine $\mathrm{LC}_{50}$ of crude cocoa powder extract. The mice were infected with $1 \times 10^{7}$ of ANKA and NK65 strains of Plasmodium berghei intraperitoneally, while graded doses of the extract were administered by an intra-gastric intubation based on the body weight of mice. Blood samples were analyzed for microscopy and flow cytometry for CD4+ cell counts. Results: The onset of infection was delayed in the group treated before inoculations on day 3 and the level of $P$. berghei parasitemia was positively associated with induction of CD4+ cells while the negative control group that received normal saline had progressive increase of parasitemia. The mean survival time could not go beyond day14 in ANKA, though both strains responded to CPE in a similar way with chloroquine as a positive control. The CD4+ cells counted increased in both strains treated before and during inoculations and the episodes of malaria was suppressed compared with the control. Conclusion: This study has demonstrated that the antiplasmodial activity of CPE was associated with the level of CD4+ T-cells proliferation which initiated the protective immune response. This therefore calls for efforts to ensure adequate intake of cocoa powder to boost immunity against malaria.
\end{abstract}

Keywords: Immune Boosting Effect; Cocoa Powder Extract; Plasmodium Berghei; Antimalarial Activity; BALB/c Mice

\section{Introduction}

Falciparum malaria among other species of Plasmodium is a complex disease and a major cause of morbidity and mortality [1]. It is endemic in Sub-Saharan Africa with more than $90 \%$ of the total population at risk with more than 1 million deaths each year and also contributes indirectly to so many additional complications mainly in children and pregnant women. Malaria in Nigeria is a major cause of morbidity and mortality. It is endemic throughout the country with more than $90 \%$ of the total population at risk of stable endemic malaria. At least $50 \%$ of the population suffers from at least one episode

${ }^{*}$ Corresponding author. of malaria each year as the disease is the commonest cause of outpatient attendance across all age groups [2]. Despite some breakthrough in the synthesis of antimalarial drugs, the dream of the global eradication begins to fade with the growing number of cases of rapid spread of drug resistance in the parasite [3,4], and the serious risk of development of resistance in P. falciparum to artemisinin-based combination therapy (ACT) with the recent observation of two cases of clinical resistance in Cambodia [5-8]. Although ACT should represent the best option available to prevent the induction of resistance parasites, the risk of development of resistance in P.falciparum to artemisinin, compounds is serious, given the cases of diminished $P$. falciparum susceptibility to arthemeter [9]. 
Recently, it was observed that variations in susceptibility profiles of malaria to artemisinin deriva- tives have been associated with single nucleotide poly- morphisms (SNPs) suggesting a possible role of (pfATPase6) gene as a molecular marker of artemisinin resis- tance.

Therefore, the urgent need to develop new and effective antimalarial agents [10] is crucial since large population of people in the tropics still rely on plant-based therapy [11]. Theobroma cacao contains the highest levels of Total Antioxidant Capacity (TAC) and procyanidins [12] that led to their therapeutic effects against the development of atherosclerosis, hepato-carcinogenesis, and proliferation of human breast cancer cells $[7,13]$.

A study on cocoa polyphenols and human cytokine activation concluded that cocoa, as a potential immune modulator, may have therapeutic advantages in human disease that involve activation of the immune system [8, 14]. Oligomeric procyanidins isolated from cocoa have been shown to possess biological activities potentially relevant to oxidant defences and immune functions [15]. Flavanols kaempferol, quercetin and isoquercetrin were screened for inhibitory effects on intraerythrocytic malaria parasites [16]. The complex polyphenols in human plasma following consumption of flavanol-rich cocoa $[17$, 18] seem to be connected with less severe malaria [19] or more rapid clearance of parasitemia [20]. The complex polyphenols (procyanidins whose availability was once in doubt, have been detected in human plasma following consumption of flavanol-rich cocoa [5] seems to be connected with less severe malaria [17] or more rapid clearance of parasitemia [21].

Also, the ingestion of flavanoid-rich cocoa produced high plasma levels of Nitric Oxide (NO) which has been reviewed [22] to mediate inhibition of malaria parasites by hepatocytes and monocytes. In the complex immune response to malaria, $\gamma \mathrm{T}$ cells have been shown to have the capacity to directly inhibit the parasite growth. Both the CD4+ and CD8+ T cells play important roles in immunity to malaria, but at different stages. During the liver stage CD8+ T cell functions are important [23], and they also contribute to protection against severe malaria. The role of DCs in malaria immunity is still relatively unknown, some studies show that the maturation of human DCs are suppressed, and that their ability to activate. T-cells are reduced by iRBC [24]. The CD4+ T cells are crucial in the immunity against asexual blood stage malaria. They produce cytokines which are involved in the activation of innate immune response and the immunity to blood stage malaria is dependent on the CD4+ T cells, anti-malarial antibodies and B cells [25]. Therefore, due to these claims, our research study on the use of cocoa powder was to investigate some anecdotal reports of its antimalarial efficacy and to authenticate its capacity to boost immunity.

\section{Materials and Methods}

\subsection{Experimental Animals}

Adult Swiss albino mice of 10 - 12 weeks old weighing between $20-30 \mathrm{~g}$ were bred and kept in plastic cages with stainless steel covers at room temperature, fed with standard mouse cubes and clean tap water ad libitum.

\subsection{Malaria Parasite}

The inoculums of ANKA and NK65 strains of Plasmodium berghei (Batch number S0594596) donated by Malaria Research and Reference Repository Center (MR4) of 10801 University Boulevard, Manassas, Virginia, United States of America were collected from Drug and Molecular laboratory of Institute for Advanced Medical Research and Training (IAMRAT), College of Medicine, University of Ibadan, Nigeria were passaged as $0.2 \mathrm{ml}$ was injected intraperitoneally into each experimental mouse which contained $1 \times 10^{7}$ Plasmodium berghei infected erythrocytes.

\subsection{Cocoa Powder}

Non-alkalized cocoa powder was collected from the Crop Processing and Utilization Division of the Cocoa Research Institute of Nigeria (CRIN) Ibadan. The preliminary polyphenols and flavanoids screening was done using standard qualitative methods described by [1].

\subsection{Brine Shrimp Test (BST Assay) on Cocoa Powder Extract}

This test was conducted according to [26,27] so as to detect the cytotoxicity activity by the brine shrimp lethality bioassay. The Probit Soft Ware as described by [28] was used to determine the results of the assay.

\subsection{Experimental Design and Grouping}

The animals were divided randomly into six groups with fifteen mice per group. The grouping was done based on the average body weight of $26 \mathrm{~g}$. Different concentrations of extract, $10 \mathrm{mg}$ of chloroquine were administered by gastric intubation for three consecutive days before, during and after the inoculations. The negative control received normal saline throughout the experiment.

\subsection{Inoculation of Mice}

Cardiac blood samples from the donor mice with percentage parasitemia of $12 \%$ and $8 \%$ for NK65 and ANKA strain respectively were withdrawn using insulin syringes of $1.0 \mathrm{ml}$ gauge containing acid citrate dextrose anticoagulant (ACD). The samples were diluted with normal saline to maintain isotonic condition, such that $0.2 \mathrm{ml}$ of $1 \times 10^{7}$ Plasmodium berghei infected erythro- 
cytes.was injected intraperitoneally into each experimental mouse.

\subsection{Preparation of Smear}

Both thin and thick smears were prepared and stained with Giemsa to estimate the percentage of RBC infected with malarial parasites and the parasite densities respectively which were carefully viewed under the microscope using a high magnification $(\times 100)$ oil immersion objective.

\subsection{Determination of CD4+ T-Cell Count for Mice}

Approximately $20 \mu \mathrm{l}$ of whole blood samples in the EDTA.K $K_{3}$ anticoagulated bottles and $20 \mu \mathrm{l}$ of the CD4+ monoclonal antibody specific for mice from Santa Cruz Biotechnology, USA was mixed and incubated for 15 minutes at room temperature in the dark. About $800 \mu \mathrm{l}$ of the dilution buffer was added and mixed after completion of incubation. The count analysis for CD4+ cells was carried out and recorded with the Cyflow Machine made by Partec Co., Germany with the excitation light source of $532 \mathrm{~nm}$ (green solid state laser).

\subsection{Statistical Analysis}

The results were expressed in terms of mean \pm standard deviation (SD). Parameters in the groups were compared by one-way and univariate Analysis of Variance (ANOVA) using the computer software Statistical Package for Social Sciences (SPSS) Version 11.0. The values of treated groups were considered significantly different from that of control when p-value was less than 0.05 .

\section{Results}

Figures 1(a)-(c) showed the histogram of immune panel for CD4+ counts before and after gating of untreated infected control, effects of extract treatment of the specimen before inoculation and the level of CD4+ counts on normal uninfected control respectively. The CD4+ count of untreated control was 840 cells/ $\mu$ l. However, when treated with cocoa extract, the immune response was boosted up to 4866 cells/ $\mu$, but when compared with the level of CD4+ count on normal uninfected control, the number of cells/ $\mu$ l was 1767 (Figures 1(a)-(c)). Chloroquine was used in mice with $P$. berghei as a standard antimalarial drug and the absolute CD4+ cell count amounted to 2934 (Figure 2).

Figure 3 compares the effects of pretreatment on percentage parasitemia of mice infected with strains of $P$. berghei. The extract suppressed the onset of parasitemia to a non-detectable levels compared with CQ treatment. Percentage parasitemia for both ANKA and NK65 strains of $P$. berghei in untreated-infected control group were observed to increase remarkably as the infections progressed until death in comparison to the cocoa extract treated and chloroquine groups after the establishment of the parasites. Meanwhile, the parasite infected-treated groups with standard drug (CQ) had rapid decrease in parasitemia of ANKA strains in the course of treatment as at day7 and were observed to be greater than that of NK65 infected-treated group. It was clearly depicted in the bar chart that most ANKA strains treated with normal saline alone could not survive beyond day 14 of post infection while none of the strains survived till day 28 of post treatment days. The mean survival time (MST) of infected treated group with saline was observed to have the least value of $(9.9 \pm 4.8)$ compared with chloroquine and cocoa powder extract group with $(30.4 \pm 3.2)$ and $(34 \pm 2.8)$ respectively.

\section{Discussion}

Rational design of malaria vaccine would be the ultimate approach to the eradication of the menace globally if adequate emphasis is given to the understanding of the role of immune responses in control of malaria diseases. The CD4+ $\mathrm{T}$ cells are crucial in the immunity against asexual blood stage malaria as they produce cytokines which are involved in the activation of innate immune response, and they are also required for the B-cell production of anti-malaria antibodies as reported by Stephens et al., 2006. This study therefore investigated the role of an intact immune system in the efficacy of antimalarial chemotherapy, as this provided the scientific basis for the use of cocoa powder as an immune booster against the onset of malaria.

The results from the study showed that mice treated prior the inoculation of the lethal strains of $P$. berghei with crude extract of cocoa powder had significant delay in parasitemia build-up after 72 hours of infectivity since the percentage parasitemia in the infected untreated group was observed to increase significantly $(p \leq 0.05)$ on day 3 when compared with chloroquine and extract treated group (Figure 3). This indicates that the chemosuppression of parasitemia was due to prophylactic activity of crude aqueous extract of cocoa powder that suggests the involvement of diglycosides of flavanol which have been proven to retard the life cycle of malaria parasites [7]. This may have contributed to the longer survival period of cocoa powder extract treated group. Also, it could be in connection with the procyanidins in cocoa that kept oxidative stress due to malaria infection under control by protecting cell membranes and augumenting anti-oxidant protection of mem- brane of cells exposed to malaria parasitemia [22].

The untreated control group for both ANKA and NK65 strains of $P$. berghei had their parasitemia 


\begin{tabular}{|l|}
\hline CD4+ $[$ cells $/ \mu 1]:$ \\
\hline $\mathbf{8 4 0}$
\end{tabular}

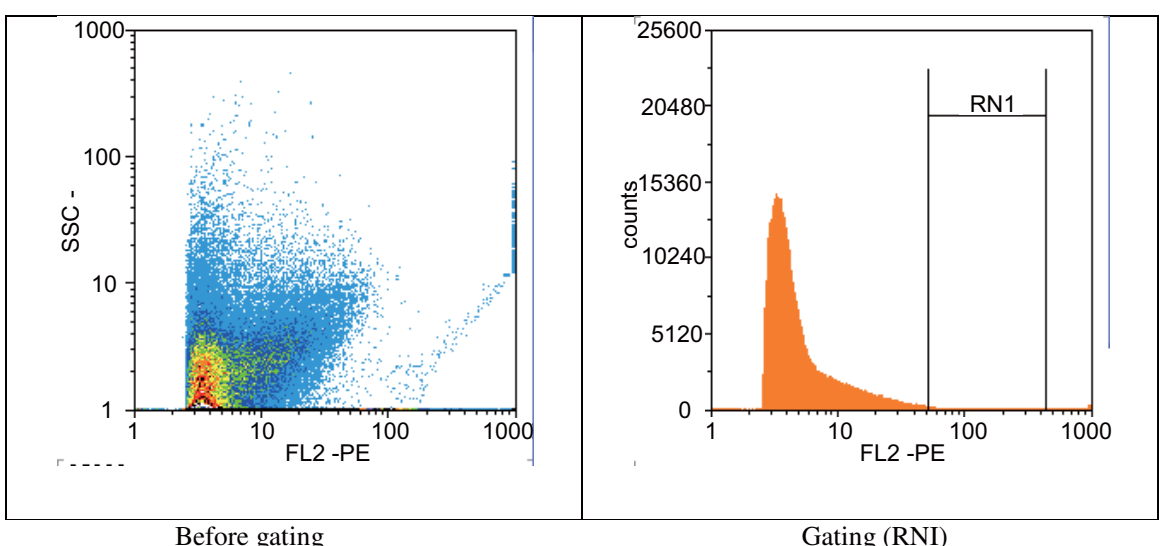

(a)

\begin{tabular}{|l|}
\hline $\mathrm{CD} 4+[$ cells $/ \mu 1]:$ \\
\hline $\mathbf{4 8 6 6}$ \\
\hline
\end{tabular}

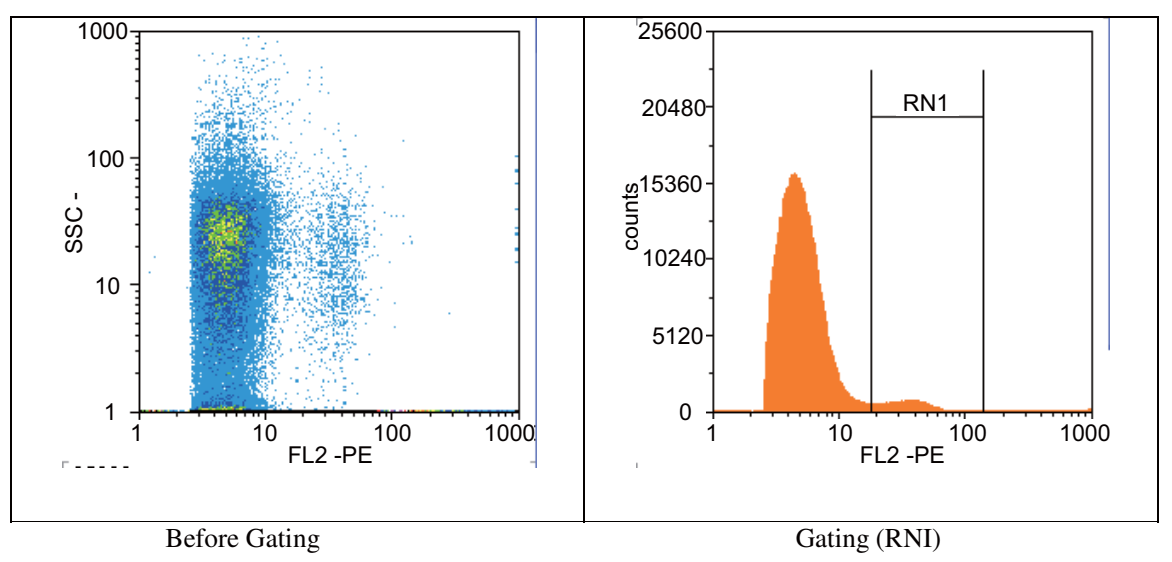

(b)

\begin{tabular}{|l|}
\hline CD4+ [cells/ $\mu 1]:$ \\
\hline $\mathbf{1 7 6 7}$ \\
\hline
\end{tabular}

Histograms:

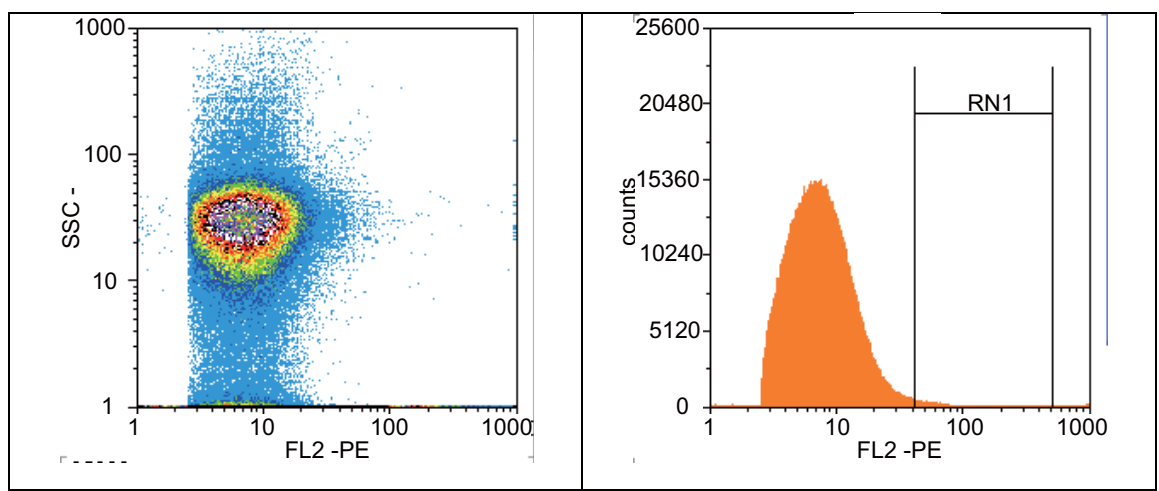

(c)

Figure 1. (a) Histogram of immune panel for CD4+counts of infected mice treated with saline. (b) The effects of extract treatment before inoculation on CD4+counts. (c) Showing the level of CD4counts on normal uninfected control. 


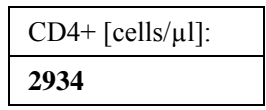

Histograms:

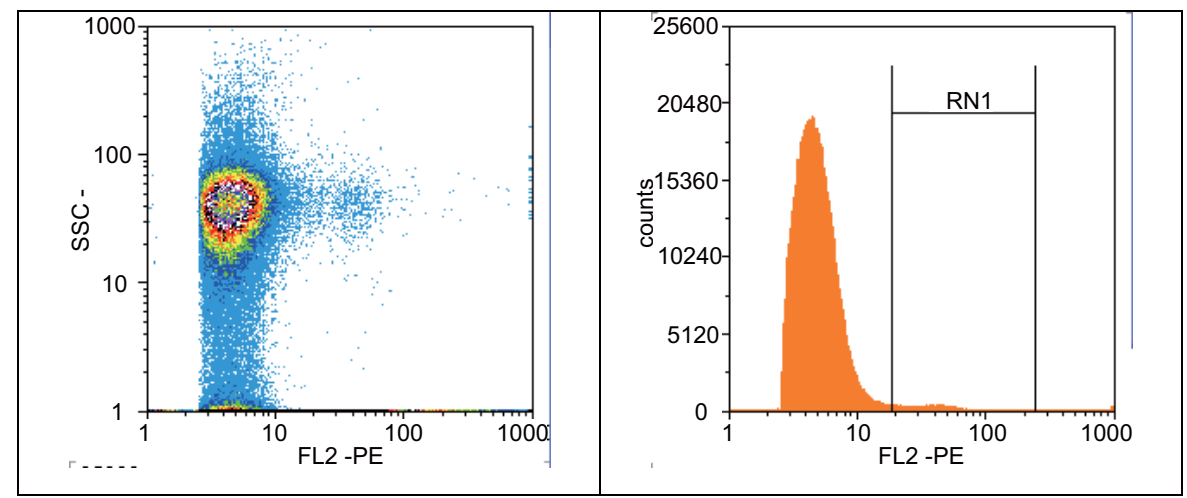

Figure 2. Showing the level of CD4 counts of mice treated with $10 \mathrm{mg} / \mathrm{ml}$ Chloroquine.

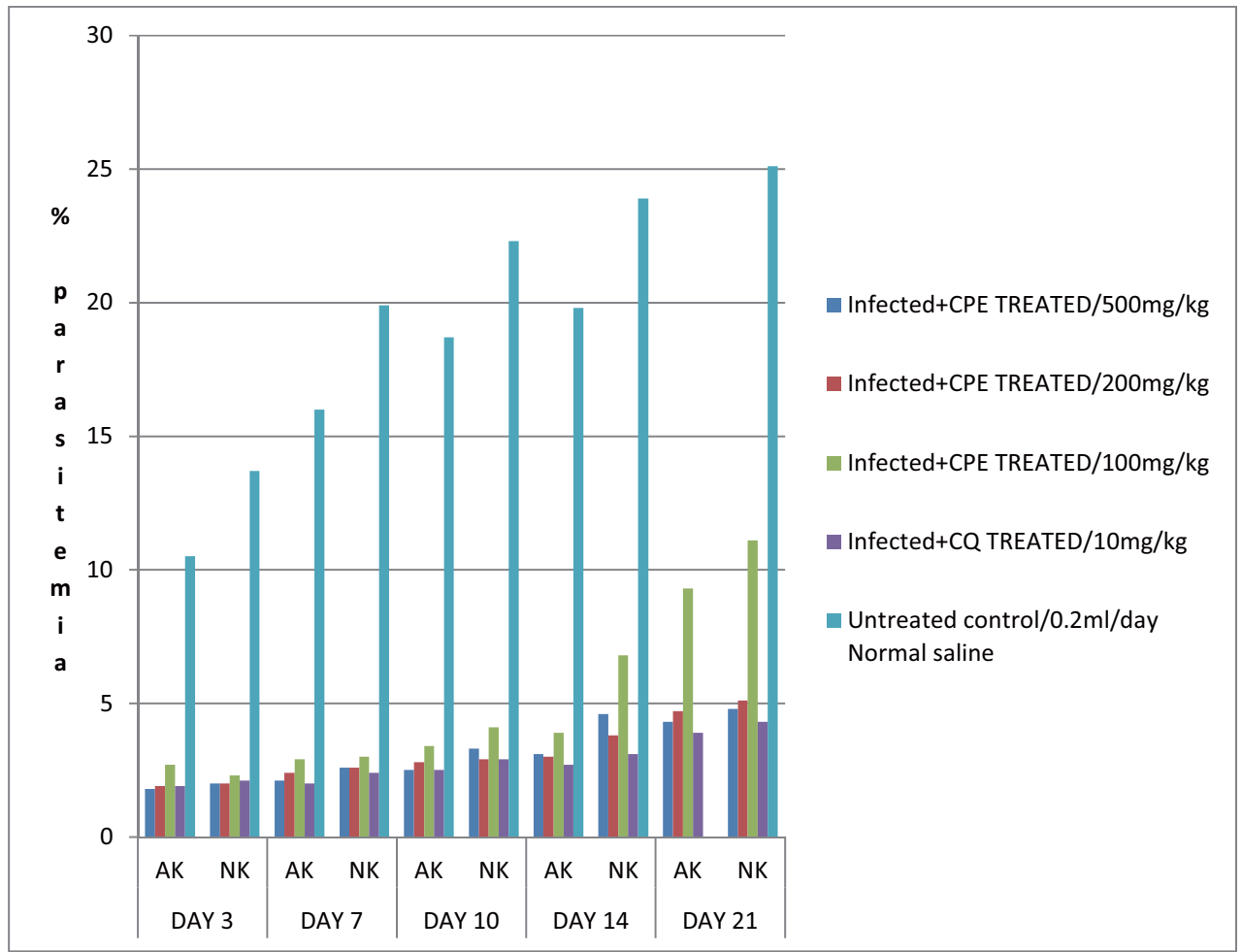

Figure 3. Effects of pretreatment on percentage parasitemia of mice infected with strains of $P$. berghei.

increased progressively until death. This may be attributed to the significant decline in the bodyweight throughout the cause of post-infection days due to severe anaemia that was observed through packed cell volume (Weatherall et al., 1983). This decrease in packed cell volume could be as a result of reducing hemo-concentration derived from decrease red blood cells count mass due to hemolysis.

In our findings, the rate of CD4+ T-cell counts were elevated among the groups treated with crude aqueous extract of cocoa powder resulting in low level of parasitemia. Interestingly, the higher the counts of CD4 cells in the treatment groups, the lower the establishment of parasite as at day 3 . This may be connected with immune boosting and humoral immunity act in concert or sequentially to control and clear a blood- stage malaria infection [29]. According to a study on cocoa poly- phenols and human cytokines that conclude cocoa as a potential immune modulator, this may have therapeutic advantages in human disease that involve activation of immune system 
$[20,30]$.

CD4+ counts in all the infected-treated groups were higher as indicated on the peaks at the gated region of the histogram of the immune panel. This is in accordance with the observation in a related study in which there was early interaction between blood stage of Plasmodium parasites and the immune system during the first few days in a malaria, gamma $\mathrm{T}$ cells expand and have been shown to have the capacity to directly inhibit the parasite growth [23]. In addition, it was also reported that immunity to blood stage malaria is dependent on the CD4+ cells [18]

Rodent models have been validated through identification of several conventional antimalarials, though primate models seem to provide better prediction of efficacy in human, nevertheless, Plasmodium berghei is an appropriate rodent parasite for the study as it has been used in the prediction of treatment outcomes and that the strains (ANKA/NK65) used are sensitive to chloroquine and this prompted the choice of the drug as a reference standard drug [31]. Our study compared the practicability of using two different strains of $P$. berghei in respect to their drug or extract susceptibility. There was a little variability in the activity of the extract against the two strains and this could be due to the lethal nature of the strains.

Furthermore, mortality of infected mice with ANKA strains of P.berghei started as early as day 14 post infection, though responded better to chloroquine. This is because the parasite invaded almost all the reticulocytes within a very short period of time when compared with day 19 post infection days in the infected mice with NK65 strains. This is in concordance with earlier study which reported that the chemo-resistance of malaria may be related to the selection of strains of rodent parasites which depend on how the merozoites penetrate into the erythrocytes either or not to maintain the infection for any length of time [32].

The results obtained from this study showed that cocoa powder extract has prophylactic antimalarial efficacy in P.berghei infected mice. Also, CD4+ helper T-cell has a protective influence against malaria infection by suppressing its proliferation when aqueous cocoa powder extract helped to boost CD4+ helper T-cell counts in $\mathrm{BALB} / \mathrm{c}$ infected with malaria parasite. This therefore provides scientific evidence that consumption of cocoa powder has protection against malaria and also suggests a possible use of cocoa powder as an immune booster against malaria.

In conclusion, as a natural remedy for malaria treatment and prevention, unsweetened cocoa powder intake should be encouraged as a daily food intake since aqueous extract of cocoa powder has shown to be efficacious against malaria infections with prophylactic effects. Mo- reover, in vivo and in vitro investigative studies with the purified fractions will be carried out in future study.

\section{Acknowledgements}

The authors would like to acknowledge the assistance of Mrs. Victoria Thomas (a laboratory assistant) of IAMRAT, College of Medicine, University of Ibadan for general cleaning and changing of animal beddings, and not forgetting Mr. Balogun of Chemistry Department, U.I. for his role in cytotoxicity assay.

\section{REFERENCES}

[1] H. O. Edeaga, D. E. Okwu and B. O. Mbaebie, "Phytochemical Constituents of Some Nigerian Medicinal Plants," African Journal of Biotechnology, Vol. 4, No. 7, 2005, pp. 685-688.

[2] Federal Ministry of Health, "Report of Malaria Situation Analysis Survey Conducted in the Six Zones of Nigeria," FMOH, Abuja, 2000.

[3] L. M. Barat and P. B. Bloland, "Drug Resistance among Malaria and Other Parasites," Infectious Diseases Clinics of North America, Vol. 11, 1997, pp. 969-987. doi:10.1016/S0891-5520(05)70400-1

[4] L. Gu, S. E. House, X. Wu, R. L. Prior, "Procyanidin and Catechin Contents and Antioxidant Capacity of Cocoa and Chocolate Products," Journal of Agricultural and Food Chemistry, Vol. 54, No. 11, 2006, pp. 4057-4061. doi:10.1021/jf060360r

[5] R. Holt, S. Lazarus, M. Sullards, Q. Zhu, D. Schramm and J. Hammerstonne, "Procyanidin Dimer B2 [EpicaTechin] in Human Plasma after the Consumption of Flavanol-Rich Cocoa," The American Journal of Clinical Nutrition, Vol. 76, No. 4, 2002, pp. 798-804.

[6] K. Kiseko, M. Hiroyuki, F. Syun-ichi, K. Tomotaka and M. Seiji, "Antimalarial Activity of Leaf-Extract of Hyrangea Macrophyla, a Common Japanese Plant," Acta Medica Okayama, Vol. 54, No. 5, 2000, pp. 227-232.

[7] N. Murakami, "Exploration for New Functions of Polyphenol Food Additives and Investigation on Structures and Safety of Their Metabolites," The Japan Food Chemical Research Foundation Research Reports No. 9, 2003.

[8] H. Noedi, Y. Se, K. Schaecher, B. L. Smith, D. Socheat and M. M. Fukuda, "Evidence of Artemisinin-Resistant Malaria in Western Cambodia," The New England Journal of Medicine, Vol. 359, No. 24, 2008, pp. 2619-2620. doi:10.1056/NEJMc0805011

[9] R. Jambou, E. Legrand, M Niang, N. Khim, P. Lim, B. Volney, M. T. Ekala, C. Bouchier, P. Esterre, T. Fandeur and O. Mercereau-Puijalon, "Resistant Plasmodium falciparum Field Isolates to in Vitro Artemether and Pointmutations of the SERCA PfATPase 6," Lancet, Vol. 366, No. 9501, 2005, pp. 1960-1963. doi:10.1016/S0140-6736(05)67787-2

[10] J. L. McLaughlin, "Methods," Journal of Plant Biochemistry \& Physiology, Vol. 6, 1991, pp. 1-32.

[11] K. Neukam, W. Stahl, H. Tronnier, H. Sies and U. He- 
inric, "Consumption of Flavanol-Rich Cocoa Acutely Increases Microcirculation in Human Skin," European Journal of Nutrition, Vol. 46, No. 1, 2007, pp. 53-56. doi:10.1007/s00394-006-0627-6

[12] D. J. Finney, "Probit Analysis," 3rd Edition, Cambridge University Press, Cambridge, 1971.

[13] I. Amin, B. K. Koh and R. Asmah, "Effect of Cacao Liquor Extract on Tumor Marker Enzymes during Chemical Hepatocarcinogenesis in Rats," Journal of Medicinal Food, Vol. 7, No. 1, 2004, pp. 7-12. doi:10.1089/109662004322984635

[14] E. Legrand, B. Volney, J. B. Meynard, P. Esterre and O. Mercereau-Puijalon, "Resistance to Dihydroartemisinin," Emerging Infectious Diseases, Vol. 13, No. 5, 2007, pp. 808-809. doi:10.3201/eid1305.061442

[15] E. Badell, C. Oeuvrary, A. Moreno, S. Soe, N. Van Rooijen and P. Druilhe, "Human Malaria in Immunocompromised Mice: An in Vivo Model to Study Defense Mechanisms against Plasmodium falciparum," Journal of Experimental Medicina Sciences, Vol. 192, No. 11, 2000, pp. 1653-1660. doi:10.1084/jem.192.11.1653

[16] M. Menegon, A. A. Talha, C. Sevenni, S. M. Elbushra, A. A. Moamedani, E. M. Malik, T. A. Mohammed, W. H. Wernzdorfer, G. Majori and B. Y. Nour, "Frequency Distribution of Antimalaria Drug Resistant Alleles among Plasmodium falciparum Isolates from Gezira State, Central Sudan, and Gedarif State, Eastern Sudan," The American Journal of Tropical Medicine and Hygiene, Vol. 83, No. 2, 2010, pp. 250-257. doi:10.4269/ajtmh.2010.09-0514

[17] G. I. Hassan, U. Gregory and H. Maryam, "Serum Ascorbic Acid Concentration in Patients with Acute falciparum Malaria Infection: Possible Significance," Brazilian Journal of Infectious Diseases, Vol. 8, No. 5, 2004, pp. 378381.

[18] R. Stephens and J. Langhorne, "Priming of CD4+ T Cells and Development of CD4+T Cell Memory; Lessons for Malaria," Parasite Immunology, Vol. 28, No. 1-2, 2006, pp. 25-30. doi:10.1111/j.1365-3024.2006.00767.x

[19] J. B. Harborne, "Phytochemical Methods: A Guide to Modern Techniques of Plant Analysis," 3rd Edition, Chapman and Hall, London, 1998.

[20] T. K. Mao, "Effect of Cocoa Flavanols and Their Related Oligomers on the Secretion of Interleukin-5 in Peripheral Blood Mononuclear Cells," Journal of Medicinal Food, Vol. 5, No. 1, 2002, pp. 17-22. doi:10.1089/109662002753723188

[21] A. Metzger, G. Mukasa, A. H. Shankar, G. Ndeezi, G. Melikian and R. D. Semba, "Antioxidant Status and Acute Malaria in Children in Kampala, Uganda," The American Journal of Tropical Medicine and Hygiene, Vol. 65, No. 2, 2001, pp. 115-119.
[22] D. J. Weatherall, S. Abdalla and M. J. Pippard, "The Anaemia of Plasmodium falciparum Malaria," Ciba Foundation Symposium, Vol. 94, 1983, pp. 74-97.

[23] E. Nardin, F. Zavala, V. Nussenzweig and R. S. Nussenweig, "Pre-Erythrocytic Malaria Vaccine; Mechanisms of Protective Immunity and Human Vaccine Trials," Parassitologia, Vol. 41, No. 1-3, 1999, pp. 439-402.

[24] B. C. Urban and N. M. Stevenson, "Early Interactions between Blood-Stage Plasmodium Parasites and the Immune System," Current Topics in Microbiology and Immunology, Vol. 297, 2005, pp. 25-70. doi:10.1007/3-540-29967-X_2

[25] Q. Y. Zhu, D. D. Schramm, H. B. Gross, R. R. Holt, S. H. Kim and T. Yamaguchi, "Influence of Cocoa Flavanols and Procyandins on Free Radical-Induced Human Erythrocyte Hemolysis," Clinical and Developmental Immunology, Vol. 12, No. 1, 2005, pp. 27-34. doi:10.1080/17402520512331329514

[26] B. N. Meyer, N. R. Ferrigni, J. E. Putman, L. B. Jacobson, D. E. Nichols and J. L. McLaughlin, "In Vivo Lethality in BST Assay," Planta Medica, Vol. 45, No. 5, 1982, pp. 3134. doi:10.1055/s-2007-971236

[27] C. Guinovart, M. M. Navia, M. Tanner and P. L. Alonso, "Malaria: Burden of Disease," Current Molecular Medicine, Vol. 6, No. 2, 2006, pp. 137-140. doi: $10.2174 / 156652406776055131$

[28] D. Dawit, M. Eyassu, D. Asfaw, A. Dawit, U. Kelbessa, M. Wallenlign, M. A. Daniel, M. Yared, "In Vivo Antimalarial Activity of Hydroalcoholic Extracts from $\mathrm{As}$ paragus africanus Lam. In Mice Infected with Plasmodium berghei," Ethiopian Journal of Health Development, Vol. 2, 2006, pp. 112-118.

[29] J. Langhorne, "The Role of $\mathrm{CD}^{+}{ }^{+} \mathrm{T}$-Cells in the Immune Response to Plasmodium chabaudi," Parasitology Today, Vol. 5, 1989, pp. 362-364.

doi:10.1016/0169-4758(89)90113-0

[30] R. Ramasamy, "Molecular Basis for Evasion of Host Immunity and Pathogenesis in Malaria," Biochimica et Biophysica Acta, Vol. 1406, No. 1, 1998, pp. 10-27. doi:10.1016/S0925-4439(97)00078-1

[31] A. F. David, J. R. Phillip, L. C. Simon, B. Reto and N. Solomon, "The Antimalarial Drug Discovery: Efficacy Models for Compound Screening," Nature Reviews, Vol. 3, No. 6, 2004, pp. 509-520.doi:10.1038/nrd1416

[32] A. Beaute-Lafitte, V. Altemayer-Caillard, F. Gonnet-Gonzalez, L. Ramiaramana, A. G. Chabaud and I. Laudau, "The Chemosensitivity of the Rodent Malarias-Relationships with the Biology of Merozoites," International Journal for Parasitology, Vol. 24, No. 6, 1994, pp. 981-986. doi:10.1016/0020-7519(94)90163-5 